\title{
Precision and Accuracy in Tandem Mass Spectrometry Measurements: A Kinetics-Based Protocol for Instrument-Independent Measurements of Collision-Activated Dissociation in RF-Only Quadrupoles
}

\author{
Richard I. Martinez \\ Chemical Kinetics Division, National Institute of Standards and Technology, ${ }^{*}$ Gaithersburg, Maryland, USA \\ (J Am Soc Mass Spectrom 1990, 1, 272-273)
}

$\mathrm{T}$ landem mass spectrometry (MS/MS) is widely used for the analysis of multicomponent mixtures (e.g., targeted-component analysis and functional-class analysis) and for structure elucidation [1]. The analysis makes use of the collision-activated dissociation (CAD) of precursor ions.

The most common mode of MS/MS operation is the daughter ion scan under multiple-collision conditions, with the key MS/MS parameters selected to suit the requirements of each individual operator. For reasons enumerated in ref. 2 (and ref. 9 therein), this mode of operation produces instrument-dependent CAD spectra (which cannot be used for interlaboratory comparisons or for intralaboratory comparisons among different instruments) and can produce nonreproducible results even within a single instrument.

This was clearly demonstrated in a 1983 international round robin [3] wherein very different $C A D$ spectra were observed for the same molecule. That is, the relative intensities measured in different MS/MS instruments for any given pair of progeny ions differed by factors ranging into the hundreds, even though the same nominal operating conditions were supposedly used in each of the MS/MS instruments of the XqQ type [where $q$ denotes an rf-only quadrupole collision cell, $Q$ denotes a quadrupole mass filter, and $X$ (the first mass analyzer) can be either a $Q$ or a sector analyzer (the second mass analyzer is a $Q$ )]: for example, $Q q Q, B E q Q$ hybrid, etc. Therefore, until now it has not been possible to use a CAD spectrum of a given species (measured in one $\mathrm{XqQ}$ instrument) to identify and quantitate that same species in a different $\mathrm{XqQ}$ instrument. Notwithstanding this fact, there are currently more than $400 \mathrm{XqQ}$ instruments worldwide (representing a capital investment of more than

*NIST; formerly National Bureau of Standards.

Address reprint requests to Dr. Richard I. Martinez, Chemical Kinetics Division, National Institute of Standards and Technology, Gaithersburg, MD 20899.
$\$ 170$ million), and the total number of users is growing rapidly.

The main goal of this communication, therefore, is to provide a "public service announcement" to alert new and prospective users of MS/MS within the broadbased analytical community to the key issues and problems that must be considered with respect to precision and accuracy in $\mathrm{XqQ}$ measurements.

A kinetics-based measurement protocol [2] has been developed at NIST [sponsored by the U.S. Environmental Protection Agency (EPA)] to provide a basis for precise and accurate $( \pm 10 \%$ ) instrument-independent measurements within XqQ instruments. The NIST protocol was validated by the recent NIST-EPA International Round Robin [4], which indicated that at least half of the commercial $\mathrm{QqQ}$ instruments in the field can provide an instrument-independent representation when our kinetics-based measurement protocol is used. (The round robin also demonstrated that the symmetric charge transfer reaction ${ }^{36} \mathrm{Ar}^{+}+$ ${ }^{40} \mathrm{Ar} \rightarrow{ }^{36} \mathrm{Ar}+{ }^{40} \mathrm{Ar}{ }^{+}$can be used as a generic "transfer" standard to provide an in situ calibration of the effective target thickness in the collision region of an $\mathrm{XqQ}$ instrument $[4,5]$.)

Consequently, it is now possible to use a "standardized" CAD spectrum (a CAD spectrum of a known species measured in one $\mathrm{XqQ}$ instrument by using the NIST protocol) to identify that same species in a different $\mathrm{XqQ}$ instrument. To do so, the key MS/MS parameters (enumerated in ref. 9 of Martinez [2]) must be properly selected as detailed in ref 2 to obviate discrimination effects against product ions because of (1) reaction-induced mass discrimination within the $\mathrm{rf}$ only quadrupole collision cell, (2) the kinetic energy of CAD fragment ions entering the second mass analyzer (MSII), and (3) the intrinsic mass discrimination within MSII. [The precepts of the NIST protocol should also be applicable to other types of tandem mass spectrometers that have strong focusing properties (e.g., quadrupole-hexapole-quadrupole instru- 
ments), as long as the energy range used is the same as for $\mathrm{XqQ}$ instruments.]

Intrinsic to the NIST protocol are two key provisions intended to obviate the lack of reproducibility of CAD spectra that has been attributed to differences in the precollision internal excitation of the parent (precursor) ion undergoing $\mathrm{CAD}$ (see item 10 of section 4 in ref. 9 of ref. 2). One of these provisions is the use of $70-\mathrm{eV}$ electron ionization (EI) to prepare the precursor ion (and to ensure compatibility with existing reference libraries of EI mass spectra). Another key provision of the NIST protocol is that one must make appropriate corrections for fragment ion signals observed in the absence of the CAD target gas (see note for step $h$ on page 287 of ref 2; this would necessitate that one exclude from the CAD spectrum any fragment ions resulting from unimolecular fragmentations $[6,7])$. Thus, the NIST protocol can be used with 70 eV EI to provide long-term, instrument-independent reproducibility of CAD spectra among several $\mathrm{XqQ}$ instruments. Hence, by using $70-\mathrm{eV}$ EI, the NIST protocol [2] can be used for the development of a standardized, instrument-independent MS/MS database (or library) for $\mathrm{XqQ}_{\mathrm{Q}}$ instruments. Moreover, since the precepts of the NIST protocol address primarily the selection of the key MS/MS instrumental parameters (see above), it should be possible to use the NIST protocol with any other single ionization mode (with constant settings) to provide long-term reproducibility of $C A D$ spectra within a single $\mathrm{XqQ}$ instrument.

One of the goals of this note, therefore, is to promote discussion about the CAD database format(s) best suited to the needs of the MS/MS community (and prospective users) concerned with real-world analytical applications that involve structure identification via library matching of some kind (e.g., characterization of unknown compounds in complex mixtures of toxic materials at Superfund hazardous waste sites).

Consequently, we have proposed [2] the development of a database composed of standardized CAD spectra of EI fragment ions (fragment ions formed within the EI source by dissociative ionization of a neutral compound; cf. ref. 7). The advantages of having such a database are:

1. Standardized CAD spectra of EI fragment ions can be correlated with the neutral substructures of both known and unknown species [8]. Therefore, one can assign the structure of an unknown species on the basis of the standardized CAD spectra of EI fragment ions that correspond to the constituent neutral substructures of the unknown (as long as the unknown species contain neutral substructures for which the standardized CAD spectra of the corresponding EI fragment ions have been measured). The use of MS/MS in this way would be analogous to the use of group frequencies for identifying unknown compounds by the use of infrared spectroscopy.
2. Characterization of an unknown compound in the manner described does not require that the compound be in the database. By contrast, to characterize an unknown by spectral matching within a "library," the compound must usually be in the "library." In this regard, development of a database of standardized CAD spectra of EI fragment ions corresponding to all neutral substructures (or a subset thereof) would be very much more tractable than the development of a library of CAD spectra for all the source compounds that contain all the substructures (or a subset thereof). Consider the simplistic analogy where compounds correspond to words, and substructures correspond to letters; more than 500,000 words (compounds) can be composed with only 26 letters of the alphabet (hundreds of standardized CAD spectra of EI fragment ions).

3. The format is compatible with its use in expert systems. This should facilitate rapid real-time analysis of unknowns within computer-controlled field instruments.

4. End users are involved directly in its evolution by using critically evaluated standardized CAD spectra already in the database and by submitting new ones for inclusion in the database.

If members of the MS/MS community were to use the NIST protocol [2] to generate and contribute instrument-independent CAD spectra of species studied during the course of their work, such contributed CAD spectra (to be sent to me; see address on title page of this communication) would be included in the NIST-EPA Standardized CAD Database currently being developed in our laboratory. The database will be disseminated by NIST to the MS/MS community.

In summary, a kinetics-based measurement protocol can provide accuracy and precision for CAD measurements within $\mathrm{XqQ}$ tandem mass spectrometers. The NIST protocol can be used to develop an instrumentindependent MS/MS database (or library) for $\mathrm{XqQ}$ instruments.

\section{References}

1. Busch, K. L.; Glish, G, L.; McLuckey, S. A. Mass Spectrometry/Mass Spectrometry: Techniques and Applications of Tandem Mass Spectrometry: VCH: New York, 1988.

2. Martinez, R. I. J. Res. Nath. Inst. Std. Technol. (U.S.) 1989, 94, 281 , and all references therein.

3. Dawson, P. H.; Sun, W.-F. Int. J. Mass Spectrom. Ion Processes 1983/1984, 55, 155.

4. Martinez, R. I. Rapid Commun. Mass Spectrom. 1989, 3, 127.

5. Dheandhanoo, S. Rapid Commun. Mass Spectrom. 1988, 2, 266.

6. McLafferty, F. W.; Bente, P. F., III; Kornfeld, R.; Tsai, S.-C.; Howe, I. J. Am. Chem. Soc. 1973, 95, 2120.

7. McLafferty, F. W.; Kornfeld, R.; Haddon, W. F.; Levsen, K; Sakai, I.; Bente, P. F., III; Tsai, S.-C.; Schuddemage, H, D. R. J. Am. Chem. Soc. 1973, 95, 3886.

8. Martinez, R. 1.; Ganguli, B. Rapid Commun. Mass Spectrom. $1989,3,377$. 